Solar Physics

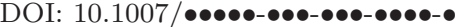

\title{
Evidence of Magnetic Helicity in Emerging Flux and Associated Flare
}

\author{
R. Chandra · B. Schmieder • G. Aulanier • \\ J.M. Malherbe \\ (C) Springer $\bullet \bullet \bullet \bullet$
}

\author{
R. Chandra • B. Schmieder • G. Aulanier - J.M. Malherbe \\ Observatoire de Paris, LESIA, UMR8109 (CNRS), F-92195 \\ Meudon Principal Cedex, France \\ R. Chandra \\ email: chandra.ramesh@obspm.fr \\ B. Schmieder \\ email: brigitte.schmieder@obspm.fr \\ G. Aulanier \\ email: guillaume.aulanier@obspm.fr \\ J.M. Malherbe \\ email: jean-marie.malherbe@obspm.fr \\ Uttarakhand Department of Science and Technology C/O
}

R. Chandra

Office of the District Magistrate, Nainital, India
\end{abstract}

Abstract The aim of this paper is to look at the magnetic helicity structure of an emerging active region and show that both emergence and flaring signatures are consistent with a same sign for magnetic helicity. We present a multi-wavelength analysis of an M1.6 flare occurring in the active region NOAA 10365 on 27 May, 2003 , in which a large new bipole emerges in a decaying active region. The diverging flow pattern and the "tongue" shape of the magnetic field in the photosphere with elongated polarities are highly suggestive of the emergence of a twisted flux tube. The orientation of these tongues indicates the emergence of a flux tube with a right hand twist, i.e. positive magnetic helicity. The flare signatures in the chromosphere are ribbons observed in $\mathrm{H} \alpha$ by the MSDP spectrograph in the Meudon solar tower and in $1600 \AA$ by TRACE. These ribbons have a 'J' shape and are shifted along the inversion line. The pattern of these ribbons suggests that the flare was triggered by magnetic reconnection at coronal heights below a twisted flux tube of positive helicity, corresponding to that of the observed emergence. It is the first time that such a consistency between the signatures of the emerging flux through the photosphere and flare ribbons is clearly identified in observations. Another type of ribbons observed during the flare at the 
periphery of the active region by the MSDP and SOHO/EIT are related to the existence of a null point, which is found high in the corona in a potential field extrapolation. We discuss the interpretation of these secondary brightenings in terms of the "breakout" model and in terms of plasma compression/heating within large-scale separatrices.

\section{Introduction}

It is believed that flare energy originates from the free energy stored in nonpotential magnetic structures. Magnetic reconnection is considered to play an important role for the sudden release of energy in solar flares. Several conditions such as: flux emergence, high magnetic field gradient, flux cancellation etc. are commonly found near flare locations.

Magnetic complexity plays an important role for strong solar activity, as studied by many authors (i.e. Schmieder, and van Driel-Gesztelyi, 2005 ; Sammis, Tang, and Zirin, 2000; Antiochos, DeVore, and Klimchuk, 1999). The magnetic complexity is commonly due to slow or fast evolution of the magnetic configuration. Shear and flux emergence are responsible of such changes in the magnetic field. Hagyard, Venkatakrishnan, and Smith (1990) have shown that the magnetic field in flaring locations is strongly sheared. Large scale shear in the magnetic field can be built up through the slow motion of footpoints stretching the length of loops. This evolution can progress through stable field configurations (Roudier et al., 2008). However, magnetic shear alone is not a sufficient condition. Flux emergence has long been considered as an important condition to trigger solar flares (Heyvaerts, Priest, and Rust, 1977; Martin et al., 1982; Shibata, Nozawa, and Matsumoto, 1992; Schmieder et al., 1994; Choudhary, Ambastha, and Ai, 1998; Chen and Shibata, 2000; Zhang, Zhang, and Zhang, 2008 and references therein). A new magnetic flux tube emerges from below the photosphere, interacts with the existing flux tubes, and the reconnection occurs in the current sheet, which forms between the old and new fluxes (Heyvaerts, Priest, and Rust, 1977). The magnetic flux may emerge already twisted (Leka et al., 1996). Sub-photospheric motions are the drivers of free energy storage and the emerging flux provides the trigger mechanism for impulsive energy release (Zhao and Kosovichev, 2004; Mason et al., 2006).

Magnetic helicity is a parameter indicating the degree of twist and writhe in an active region (see the reviews by Démoulin, 2007; Démoulin and Pariat, 2009). The helicity pattern of the Sun has characteristic features governed by an hemispheric rule: positive/negative magnetic helicity is common in the South/North hemisphere. Pevtsov (2002) summarized the different possible signatures of magnetic helicity observed on the Sun: sunspot whorls, filament chirality, sigmoids, flare ribbons. Appearance of magnetic "tongues" in an emerging active region as first discussed by López Fuentes et al. (2000) is an important signature, which tells about the helicity sign of the active region. We will use these proxies to derive the magnetic helicity of our active region. Sometimes there are mixed magnetic helicity zones in the active region. Therefore it is not always necessary 
that the helicity sign be the same in all the observational features (Green et al., 2007; Chandra et al., 2009).

The analysis of magnetic topology of an active region gives us information on possible locations of magnetic reconnection: null point (locations where magnetic field vanishes), separatrices (which separate different connectivity domain), Quasi-separatrices layers (QSL) (the region where there is a drastic change in field line linkage). QSL is a generalization of this concept proposed by Priest and Démoulin (1995) and applied to the modeling of ' $J$ ' shaped two-ribbon flares by Démoulin, Priest, and Lonie (1996).

In this paper, we study the evolution and magnetic configuration of the M1.6 flare that occurred in the NOAA 10365 active region on 27 May, 2003, using multiwavelength observations. We present the instrumentation and observations in Section 2. Section 3 describes the evolution of the magnetic field in the active region. The description of the flare at different wavelengths is presented in Section 4. In Section 5 we explain the relationship between the magnetic helicity of the twisted emerging flux tube and the magnetic reconnection leading to the M1.6 flare with 'J' shape main ribbons. In Section 6, we show the results of a linear force-free field (lfff) model of the large scale active region configuration and provide an interpretation to the presence of semicircular brightenings (secondary ribbons) in its periphery. We finally conclude that it is the first time that signatures of magnetic helicity in the photosphere (presence of tongues in longitudinal magnetic field maps), in the chromosphere (' $\mathrm{J}$ ' shaped ribbons) and in the corona (hard X-ray sources) are consistent with the existence of an emerging twisted magnetic flux tube and reconnection in the corona.

\section{Instrumentation and Observations}

The M1.6 flare occurring in the active region NOAA 10365 located S08 W10 on 27 May, 2003 was the target of an international campaign. This flare is associated with a coronal mass ejection (CME) observed by Large Angle Spectroscopic Coronagraph (LASCO, Brueckner et al., 1995) at 06:50 UT. The complex morphology of the ribbons observed at the Meudon solar tower was intriguing and initiated this study.

Figure 1 shows the temporal evolution of the flare observed by GOES 12. The eruption onset starts at 05:40 UT with a flux of $3.5 \times 10^{-6} \mathrm{Wm}^{-2}$. The maximum of flux is reached around 06:15 UT with a value in X-rays $(1-8 \AA)$ of $1.6 \times 10^{-5}$ $\mathrm{W} \mathrm{m} \mathrm{m}^{-2}$. This is a long duration event that ended around 10:00 UT.

Figure 2 presents the temporal evolution of the flare in hard X-rays (HXR) observed by Reuven Ramaty High-Energy Solar Spectroscopic Imager (RHESSI). The time profiles show that the X-ray emissions above $25 \mathrm{keV}$ are very low. The time profiles indicate that the thermal component is dominant in the flare. The two low energy band emissions slightly increase after 05:40 UT until 06:15 UT, with an impulsive event at 06:04 UT detectable in the higher energy band (25-50 $\mathrm{keV})$.

In this study, we use data from Solar and Heliospheric Observatory (SOHO)/ Michelson Doppler Imager (MDI) (time cadence of $96 \mathrm{~min}$ and pixel size of 1.98"; 


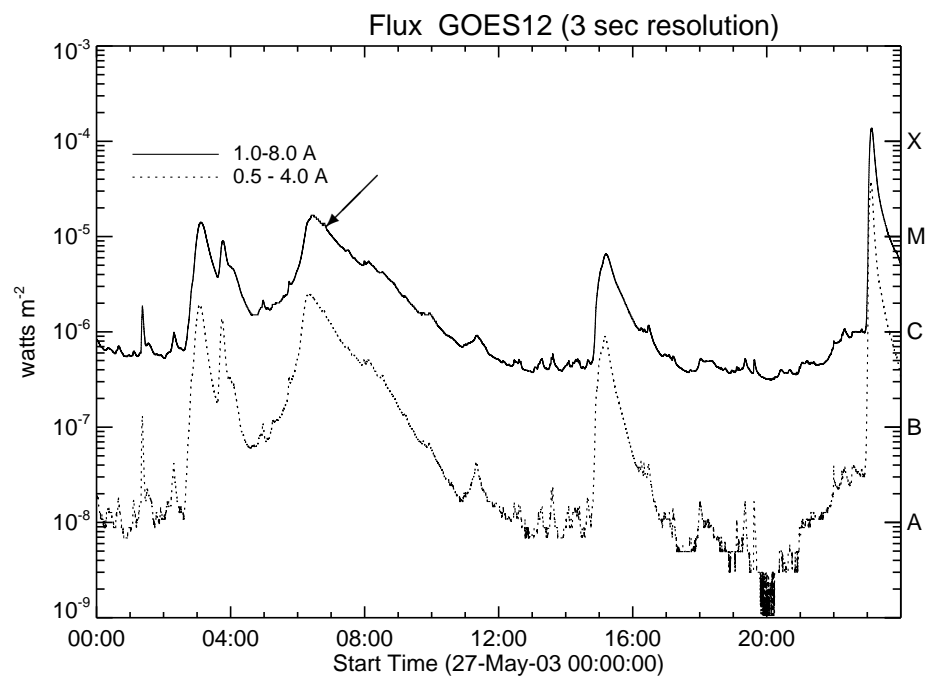

Figure 1. Temporal Evolution of the flare in GOES 12 X-ray (0.5-4) $\AA$ and (1-8) $\AA$ on May 27, 2003. The studied M1.6 flare is shown by an arrow.

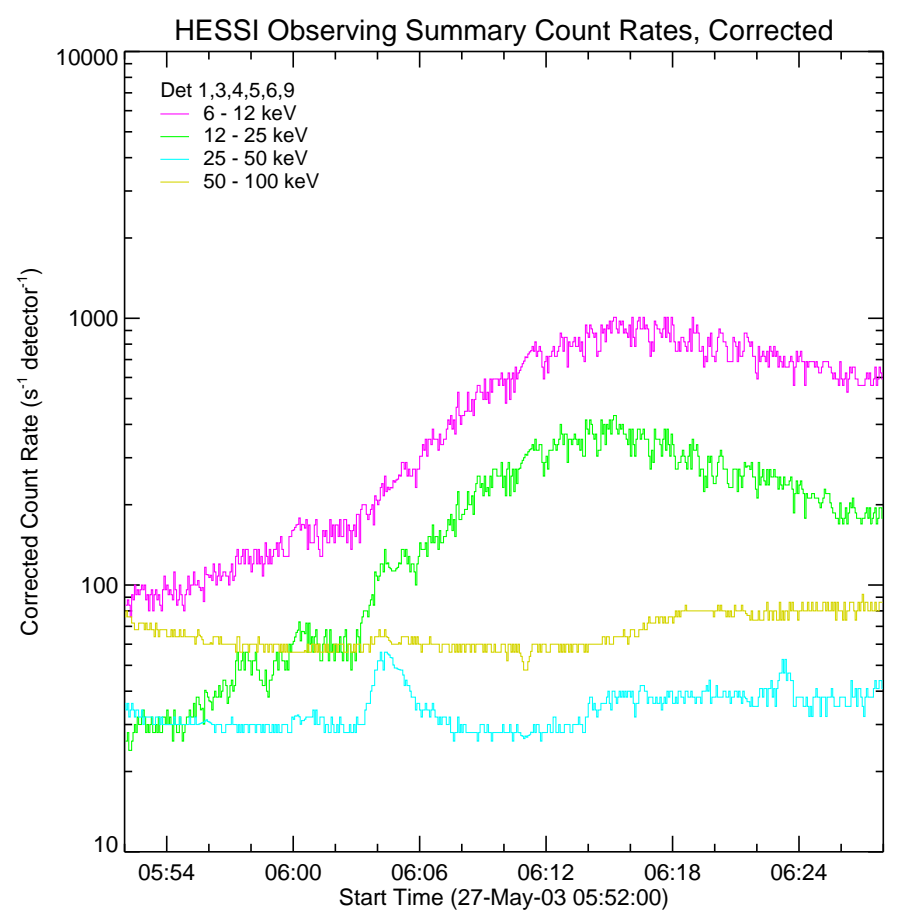

Figure 2. Temporal Evolution of the flare in HXR observed by RHESSI at 6-12, 12-15, 25-50 and 50-100 keV, respectively. 

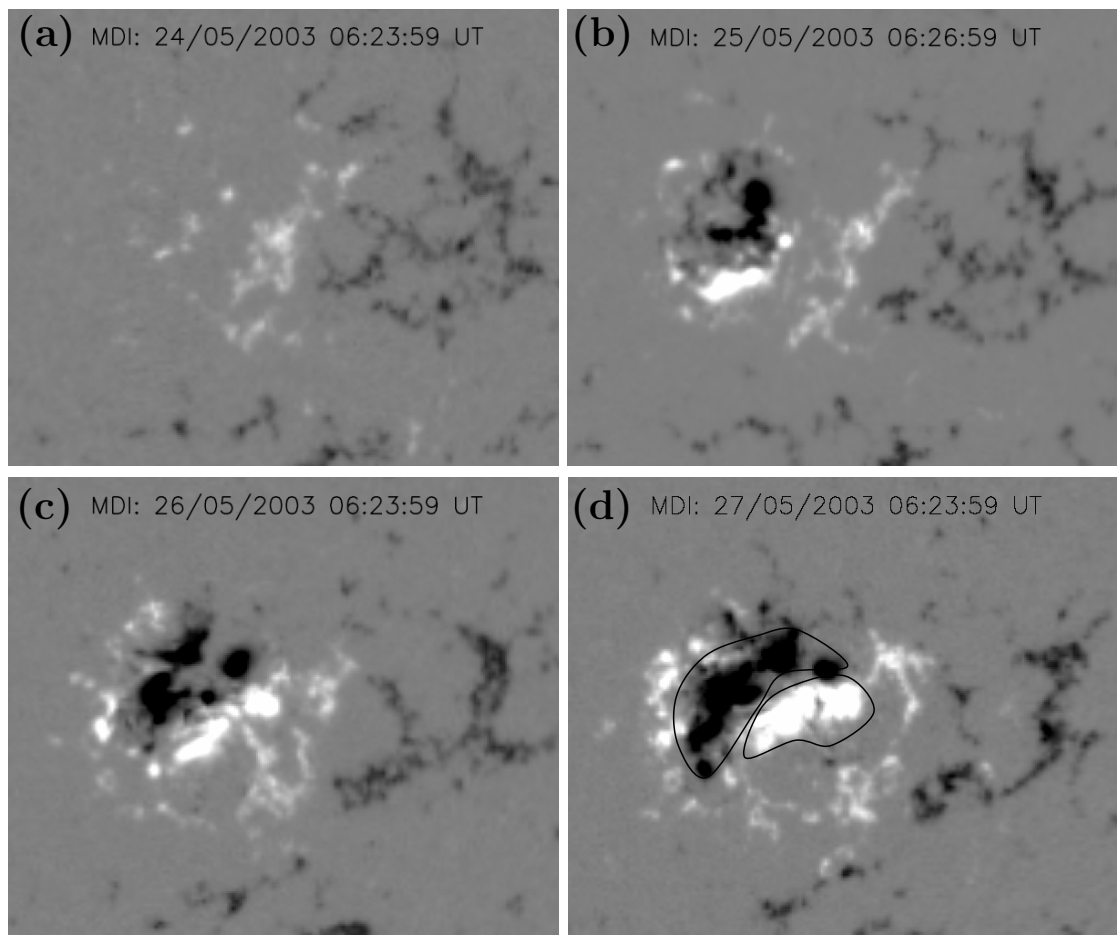

Figure 3. Evolution of the magnetic field of active region NOAA 10365. Magnetograms from 24 May, 2003 to 27 May, 2003 show the emergence of high intensity flux. The contour over the magnetogram of 27 May, 2003 shows the two magnetic tongues. The field of view of the images is $300^{\prime \prime} \times 250^{\prime \prime}$. North is up and West is to the right.

Scherrer et al., 1995 ) and EIT (time cadence of 12 min to 6 hour and pixel size of 2.5"; Delaboudinière et al., 1995), Transition Region and Coronal Explorer (TRACE) $1600 \AA$ (time cadence of 6 min and pixel size of $0.5^{\prime \prime}$, Handy et al., 1999); RHESSI (Lin et al., 2002) as well as Multi channel Subtractive Double Pass spectrograph (MSDP) of the Meudon solar tower.

The M1.6 flare has been observed in the $\mathrm{H} \alpha$ line $(6563 \AA)$ with the MSDP. The entrance slit of the spectrograph covers an elementary field-of-view of $72^{\prime \prime} \times 465^{\prime \prime}$ with a pixel size of $0.5^{\prime \prime}$. The final field-of-view of the images is $295^{\prime \prime} \times 460^{\prime \prime}$. The exposure time is $40 \mathrm{~ms}$. We performed consecutive sequences of 5 images with a cadence of 1 min. Using the MSDP technique (Mein, 1977; Mein, 1991) the field-of-view is recorded simultaneously in nine different wavelength intervals around $\mathrm{H} \alpha$, line center, separated by $0.3 \AA$. Interpolating with spline functions the observed intensity in these images, we are able to construct $\mathrm{H} \alpha$ profiles in each point of the observed field-of-view. A mean or reference disk profile is obtained by averaging over a quiet region on the disk (flat field sequence). 

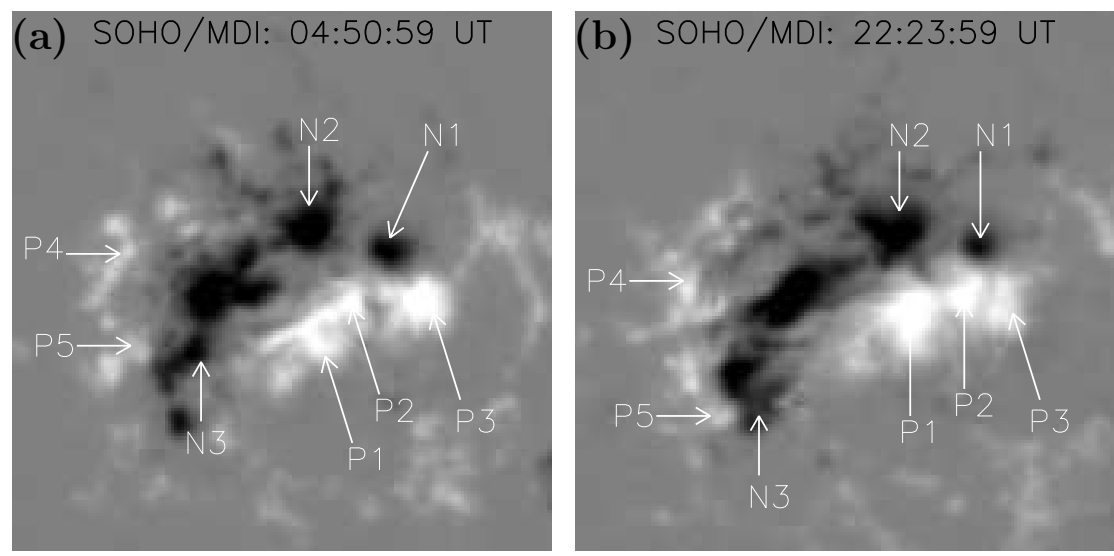

Figure 4. SOHO/MDI magnetogram of the active on 27 May, 2003 at two selected times. The polarities associated with the motions described in the text are shown by arrows ( P1, P2, P3, P4, P5: positive polarity; N1, N2, N3: negative polarity). The field-of-view of the images is $160^{\prime \prime} \times 160^{\prime \prime}$. North is up and West is to the right.

\section{Evolution of the Photospheric Magnetic Configuration}

\subsection{Global Pattern of the emerging flux}

In order to understand the cause of the flare, we need to study the magnetic configuration of the active region. Using MDI magnetograms we were able to follow its evolution during several days in May, 2003 (Figure 3). On May 24, this region had a weak field bipolar configuration with a leading negative polarity and and a diffuse following positive polarity. This corresponds to a decaying active region.

The emergence of new bipole with high intensity field occurred between May, 24 and 25 and was well visible inside the positive polarity area in the magnetograms of May 25; the bipole had a north-south orientation (the negative polarity in the north and the positive polarity in the south). Due to this continuous emergence of flux in the North part of the active region, the amplitude of the positive field increased; this positive region progressively surrounded the new emergent negative flux creating a $\delta$-spot configuration. The active region polarities rotated anticlockwise. We measure the rotation of the magnetic inversion line from May, 25 to May, 27 and find that of 25 degree. To draw the magnetic inversion line on the $\mathrm{SOHO} / \mathrm{MDI}$ magnetograms, we plot the simplified contour of zero magnetic field strength. Afterwards we measure the change in angle of the magnetic inversion line between 25 May, 2003 (06:26 UT) and 27 May, 2003 (06:23 UT).

The emergence of the new magnetic flux is a long-term process. Looking at Figure 3, between May 25 and 27, we see that the longitudinal magnetic field pattern of the emerging bipole is characterized by the existence of two elongated areas of opposite polarity labeled N1,2,3 (negative polarities) and $\mathrm{P} 1,2,3$ (positive polarities). These two areas are shifted from one another along 
Table 1. Movements of selected positive (P)/negative $(\mathrm{N})$ polarities

\begin{tabular}{lcc}
\hline Sunspot & direction & velocity $\left(\mathrm{km} \mathrm{s}^{-1}\right)$ \\
\hline P1 & northwest & 0.18 \\
P2 & northwest & 0.18 \\
P3 & northwest & 0.07 \\
P4 & northwest & 0.14 \\
P5 & south & 0.24 \\
N1 & west & 0.12 \\
N2 & west & 0.10 \\
N3 & south & 0.22 \\
\hline
\end{tabular}

the inversion line (Figure 5 (a)). This typical pattern can be explained as follows. The series of magnetograms shows the classical appearance of a bipole followed by the separation of the two opposite magnetic polarities as observed for the emergence of an untwisted $\Omega$ loop (Figure 5 (b)). An asymmetry appears in the magnetogram during the emergence of the apex of the loop because of the contribution of the azimuthal component of the emerging twisted flux tube to the observed vertical component of the photospheric field. This azimuthal component produces two elongated polarities so-called "tongues". The extension of these tongues is directly proportional to the magnitude of the twist and their position depends on the sign (positive twist in our case). The tongues are present only when the apex of the flux tube is crossing the photosphere during flux emergence. The positive/negative polarity is elongated towards the west/east with tongues. This pattern is a characteristic feature of a long-term twisted emerging flux tube as first described by López Fuentes et al. (2000) (see their Figure 5).

According to the geometry of the tongues, we conclude that the emerging tube has a right-hand twist, and consequently corresponds to a positive magnetic helicity (López Fuentes et al., 2000).

\subsection{Flow Pattern of the polarities}

We measure the speed of some selected positive (P1-P5) and negative (N1-N3) spots, which show large displacements (Figure 4). For the measurement of the location of the magnetic polarities on magnetograms we manually track the center of the each polarity. Based on the magnetograms at 04:50 UT and at 22:23 UT on May 27, we calculate the velocity and direction of the selected spots and report them in Table 1. From these measurements we see that the positive polarities $\mathrm{P} 1, \mathrm{P} 2, \mathrm{P} 3$ and $\mathrm{P} 4$ are moving towards the northwest, while polarity $\mathrm{P} 5$ is moving towards south. The negative polarity spots, i.e. N1 and $\mathrm{N} 2$ are moving towards the west, while the spot N3 is going towards south. The velocities of these spots range from 0.07 to $0.24 \mathrm{~km} \mathrm{~s}^{-1}$.

These velocities are in agreement with the local correlation tracking (LCT) analysis done by Chae, Moon, and Park (2004). The photospheric motions of these emerging spots lead to a large diverging and shearing flow pattern of 
(a)

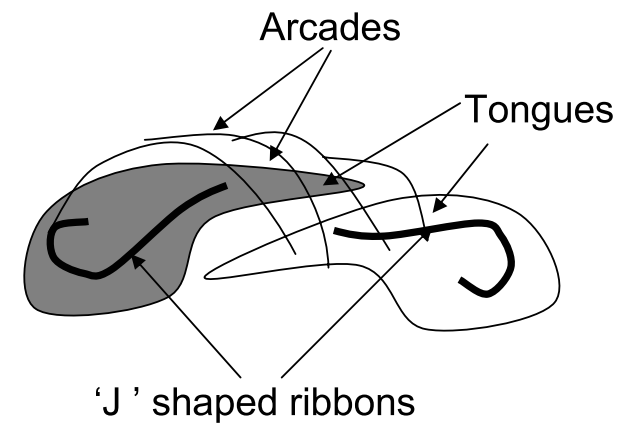

(b)

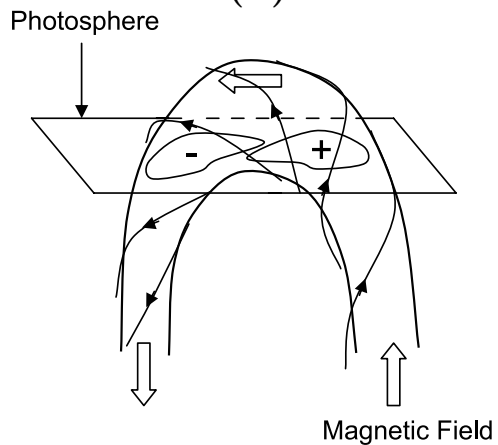

Figure 5. Sketch of the signatures of positive magnetic helicity observed in the active region NOAA 10365 during the M1.6 flare on May 272003.

the magnetic field, inducing a non-potential configuration along the magnetic inversion line. The resulting magnetic shear plays an important role in the onset of the flare.

\section{Description of the Flare}

The flare was observed in $\mathrm{H} \alpha$ and in $1600 \AA$ by TRACE presenting ' $\mathrm{J}$ ' shaped ribbons in the central part of the active region and secondary arc-shaped brightenings at the periphery of the active region, as described in Section 6:

\subsection{MAIN 'J' SHAPED RIBBONS}

The flare was observed between 06:09 UT and 06:52 UT in H $\alpha$ with the MSDP spectrograph in the solar tower of Meudon. We follow the evolution of the chromospheric ribbons in $\mathrm{H} \alpha$. In Figure 6 we show the $\mathrm{H} \alpha$ reconstructed images in the line center, indicating the different flare ribbons. The two main flare ribbons are ' $\mathrm{J}$ ' shaped. We distinguish four very bright ribbons (R1 to R4) and three secondary ribbons, less bright $\left(\mathrm{R}^{\prime} 1\right.$ to $\left.\mathrm{R}^{\prime} 3\right)$. $\mathrm{R} 2$ and $\mathrm{R} 3$ are parts of the same ribbon though not formed exactly at the same time. The pairs of ribbons R1, $\mathrm{R} 2$ and R3, R4 are part of the main flare; they are located non-symmetrically at both sides of the inversion line. At 06:15 UT, the peak time of the flaring event, some brightenings porpagated along the flare ribbons. Such a propagation has been noticed in previous flares (Berlicki et al., 2004) and may be explained by a slipping reconnection mechanism (Aulanier et al., 2007).

TRACE observed the central part of the flare in $1600 \AA$ with a cadence of 6 min. This allows us to identify the central ' $\mathrm{J}$ ' shaped ribbons, which were very similar to those seen in $\mathrm{H} \alpha$. An enlarged view of the two ' $\mathrm{J}$ ' shaped ribbons seen in $\mathrm{H} \alpha$ and TRACE $1600 \AA$ is presented in Figure 6 (b, c). We shall discuss the ' $J$ ' shaped ribbons in the context of emerging twisted flux in Section 5. 

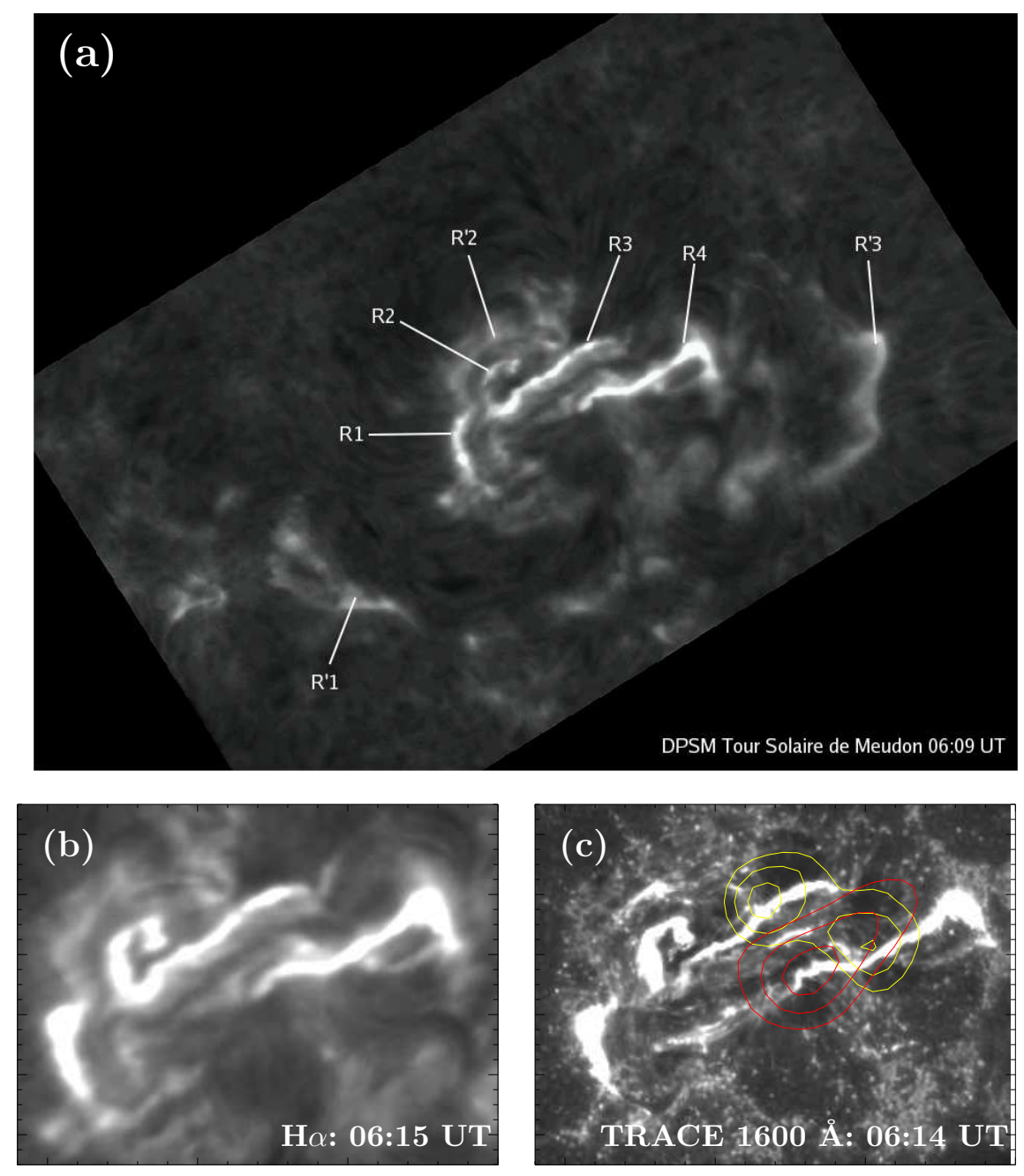

Figure 6. H $\alpha$ line-center images of the flare observed with the MSDP spectrograph of Meudon solar tower at 06:09 UT (a, field of view: $456^{\prime \prime} \times 290^{\prime \prime}$ ) and enlarged view of 'J' shaped ribbons in $\mathrm{H} \alpha$ line-center and TRACE $\left(1600 \AA\right.$ ) (b, c: field of view: $\left.160^{\prime \prime} \times 120^{\prime \prime}\right)$. The TRACE $1600 \AA$ image is overlaid by RHESSI contours (red contour: $6-12 \mathrm{keV}$ and yellow contour: $25-50 \mathrm{keV}$, contour levels: $50 \%, 70 \%$ and $90 \%$ of the peak intensity) at 06:04 UT. The North is up and West is to the right. In this Figure (a), we used the French name for the instrument.

\subsection{RHESSI IMAGES AND SPATIAL CORRELATION OF H $\alpha$, TRACE AND HXR SOURCES}

In this section we present the RHESSI images and the co-alignment of $\mathrm{H} \alpha$, RHESSI, TRACE $1600 \AA$ sources and MDI magnetogram. 

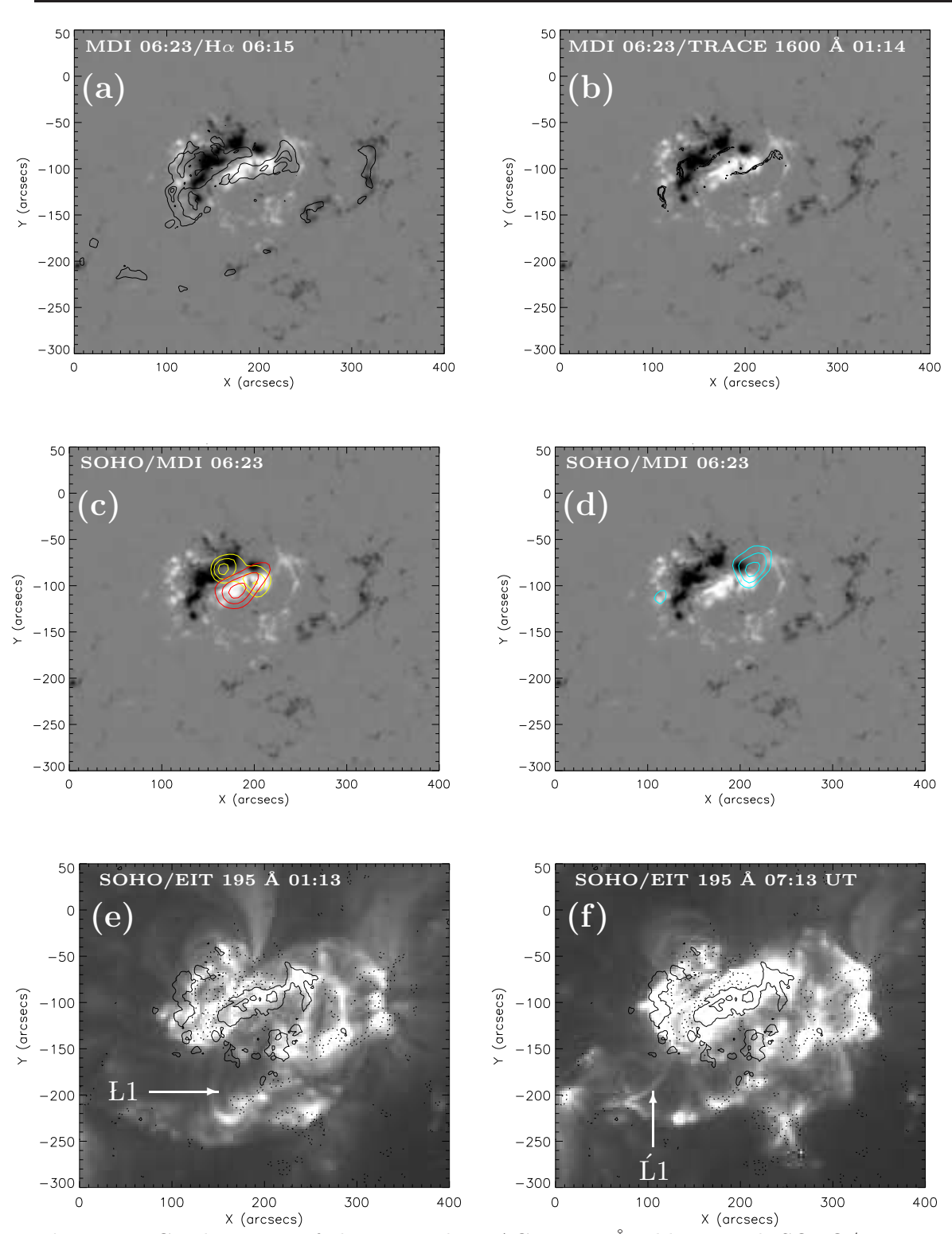

Figure 7. Co-alignment of the $\mathrm{H} \alpha$ and TRACE $1600 \AA$ ribbons with SOHO/MDI magnetogram (a, b), MDI magnetogram overlaid by RHESSI contours (6-12 keV (red contour), 25-50 $\mathrm{keV}$ (yellow contour), $15-20 \mathrm{keV}$ (cyan contour) during the flare impulsive ( 06:04 UT) and maximum phase( 06:15 UT), contour levels: $50 \%, 70 \%$ and $90 \%$ of the peak intensity) (c, d). The bottom panel presents the SOHO/EIT images before (e) and after (f) the flare onset in $195 \AA$ A. The images are overlaid by MDI magnetogram contours (solid line: positive polarity, dotted line: negative polarity, contour levels are $\pm 100, \pm 1000, \pm 1500 \mathrm{G}$ ). The loops connecting a secondary ribbon to the main flare ribbon are represented by L1 and L' 1 respectively. North is up and West is to the right. 
The co-alignment of RHESSI and MDI data was done by referring the relative position of the solar disk center of each field of view. As for the $\mathrm{H} \alpha$ and TRACE (1600 $\AA$ ) data, we compare these images with white-light images of SOHO/MDI.

We reconstructed RHESSI images in 6-12 keV, $15-20 \mathrm{keV}$ and $25-50 \mathrm{keV}$ energy channels from collimators ( $4 \mathrm{~F}$ to $9 \mathrm{~F}$ ) using the CLEAN algorithm (Hurford et al., 2002), which gives a spatial resolution of about 12 arcsec. The locations of RHESSI HXR sources in 6-12 keV and 25-50 keV overlaid on TRACE 1600 $\AA$ are shown in Figure 6 (b).

At 06:04 UT RHESSI observed two non-thermal X-ray footpoint sources in the 25-50 keV energy band, located over the main "J" shaped ribbons anchored in the positive and negative polarities (c.f. Figure 6 (b) and Figure 7). The 6$12 \mathrm{keV}$ source corresponds to a loop-top source, which looks shifted towards the south. This may be due to projection effects. During the flare peak time (06:15 UT) RHESSI observed also two HXR sources 15-20 keV, one was located at the same place as one of the previous sources (thus it could be a recurrent phenomenon) and the other one was located in the east part of the region. The new source on the left was related to the brightening of ribbons R1 and R2 at 06:15 UT.

There are several different models for the acceleration of the non-thermal electrons responsible for HXR emission in the $25-50 \mathrm{keV}$ range. Reconnection mechanism is commonly proposed. Other models include the electric field generated in a reconnection region, in shocks or turbulence. Following acceleration, electrons precipitate along the magnetic field to denser layers of the atmosphere. Electron energy loss in the chromosphere due to Coulomb collisions leads to heating, causing evaporation along the loops, while electron-ion bremsstrahlung leads to HXR emission.

Figure 7 shows the result of the co-alignment of $\mathrm{H} \alpha$, TRACE $1600 \AA$, RHESSI HXR 15-20 keV sources with MDI magnetograms. From these figures, we can infer that a strong shear is present in the flare region since the ribbon pairs R1-R2 and R3-R4 are located two-by-two in zones of opposite polarity in a non symmetric way. The loops associated with these ribbons are not in a potential state, otherwise their footpoints would be located symmetrically at both sides of the magnetic inversion line. The co-alignment permits us to explain the position of the ribbons, their shape and also how their propagation is stopped where the plages have different polarities.

\section{Magnetic Helicity of the Emerging Flux and Flare Ribbons}

The magnetic helicity is a topological quantity that measures the torsion, the shear, and the twist of magnetic field lines in a given volume (Berger, 1984). Its definition is: $\mathrm{H}=\int \mathbf{A} \cdot \mathbf{B} \mathrm{d}^{3} x$, where $\mathbf{A}$ is the potential vector of the magnetic field $\mathbf{B}$.

The magnetic helicity injection has been computed for different regions by Chae, Moon, and Park (2004) and Pariat et al. (2006) using different methods. Recently LaBonte, Georgoulis, and Rust (2007) did a statistical study relating the occurrence of big flares and found that the big flares need a high magnitude 

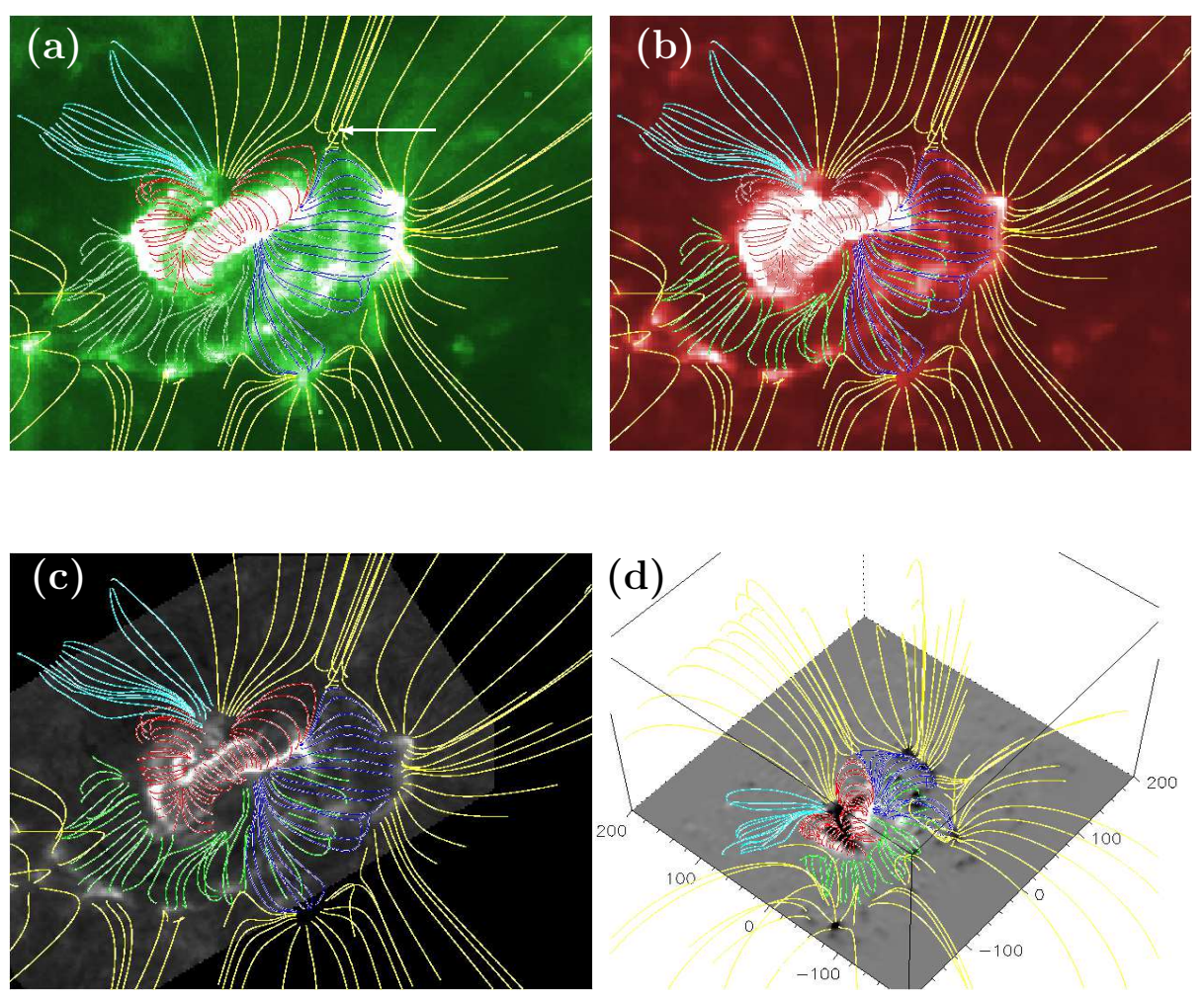

Figure 8. SOHO/EIT $195 \AA$ (07:13:30 UT; a), SOHO/EIT $304 \AA$ (07:19:17 UT; b), H $\alpha$ (06:15 $\mathrm{UT}$; c) images overlaid by magnetic field lines resulting from a potential field extrapolation. SOHO/MDI magnetogram at 06:24:11 UT and 3D view of magnetic field lines computed from a potential field model. (d). The location of null point is shown by an arrow in the upper left panel.

of helicity flux. Chae, Moon, and Park (2004) present a detailed study of the rate of helicity ejection in this active region during its passage across solar disk. The magnetic helicity of our region is found to be positive. The rate of helicity ejection peaked on 27 May, 2003, during the main activity of the active region, with the value $2.5 \times 10^{41} \mathrm{Mx}^{2}$ per hour. This is a strong evidence that the emergence of magnetic flux is an efficient process for magnetic helicity injection. The CME observed at 06:50 UT associated with this M1.6 flare very probably ejects part of this injected helicity in the heliosphere.

In Section 3 we have described in detail the emerging region. The diverging flow pattern of the emerging flux (NW for $\mathrm{Bz}<0$ and $\mathrm{SE}$ for $\mathrm{Bz}<0$, Bz being the normal component of the magnetic field), and the shape of the Bz patterns (especially in the $\mathrm{Bz}>0$ region), both are highly suggestive of magnetic "tongues" (Figure 5). The observed longitudinal magnetic field is approximately $\mathrm{Bz}$ because the region is close to the disk center. "Tongues" with such an orientation and evolution indicate the emergence of a flux tube with right hand twist, i.e. positive magnetic helicity, $\mathrm{H}>0$ (López Fuentes et al., 2000). This sign corresponds to the hemispheric helicity rule discussed by Pevtsov, Canfield, and Metcalf (1995), who 
found that $\sim 70 \%$ of southern active regions have $\mathrm{H}>0$, as the region studied by us.

In Section 4, we have shown the flare ribbons observed in $\mathrm{H} \alpha$ and in $1600 \AA$. In the central part of the region we distinguish two main forward "J" shaped ribbons (Figure 5). Such a behaviour has been already observed by Moore, Larosa, and Orwig (1995) and interpreted by Démoulin, Priest, and Lonie (1996). Using the concept of QSLs in 3D magnetic configurations, Démoulin et al. (1996); Démoulin et al. (1997) have shown that the intersection of QSLs with the chromosphere determines elongated regions that match the shape of flare ribbons. In Démoulin, Priest, and Lonie (1996), it was shown that QSLs computed in a bipolar configuration have a 'J' shape at the chromosphere. The forward ' $\mathrm{J}$ ' / reverse ' $\mathrm{J}$ ' shaped ribbons indicate that the flare is triggered by coronal reconnection below a twisted flux tube of positive/negative helicity (Démoulin, Priest, and Lonie, 1996; Williams et al., 2005) . In our case of forward 'J' shaped ribbons, the twisted flux tube should have a positive helicity. Note that this QSL interpretation is also consistent with the propagation of brightenings (see Section 4.1), in the frame of slipping or slip-running reconnection (Aulanier et al., 2007).

For the same event we find a good agreement between the magnetic helicity sign of the emerging flux and the signature of reconnection in the corona (Figure 5 ). This kind of association was never found before in a targeted study of a particular region. Williams et al. (2005) analyzed the eruption of a kink-unstable filament accompanied by a flare having 'J' shaped ribbons but did not describe the emergence phase of the region. Regarding the "tongues", only a few papers have described such phenomena (Li et al., 2007, Green et al., 2007), and they have not associated them with flare signatures.

\section{Large Scale Magnetic Field Configuration}

At the periphery of the active region 10365, secondary arc-shaped brightenings are observed at the beginning of the main flare on 27 May, 2003. These secondary ribbons are visible in $\mathrm{H} \alpha\left(\mathrm{R}^{\prime} 1, \mathrm{R}^{\prime} 3\right.$ in Figure 6$)$ and in EUV (SOHO/EIT Figure 7)

At the periphery of the active region, we observe loops in $195 \AA$ connecting these secondary ribbons with the central part of the region and it is clear that they changed their directions during the flare (see the direction of loop L1 before the flare and the direction of loop $\mathrm{L}^{\prime} 1$ after the flare)(Figure $7(\mathrm{e}, \mathrm{f})$. The secondary ribbons do not belong to the main flare, they correspond to the loops connecting relatively stable polarities of the network.

Active region NOAA 10365 is highly sheared close to the main inversion magnetic line. However, at large-scales in the active region we infer that the corona is in a near potential state, as is frequently observed far from magnetic inversion lines (Schmieder et al., 1996).

The $\mathrm{H} \alpha$ and EUV secondary ribbons can be interpreted by means of a topological analysis of the coronal magnetic field above the active region NOAA 10365 calculated from a lfff model $\nabla \times \mathbf{B}=\alpha \mathbf{B}$. For simplicity, we used a potential field model, with $\alpha=0$. This approximation is justified since we are dealing with 
fields that are far from the main current-carrying emerging and erupting twisted fields, the latter can not be modelled in this approximation. Therefore we do not model the ' $\mathrm{J}$ '-shaped ribbons.

Magnetic extrapolations in a potential configuration show the existence of null points, separatrices, larger quasi-separatrix layers at high altitudes that could be responsible for the brightening of the secondary ribbons (Priest and Démoulin, 1995; Démoulin, Priest, and Lonie, 1996; Démoulin et al., 1997). The lfff extrapolation method is the one used in the database of FRench Online MAGnetic Extrapolations (FROMAGE). It is based on the decomposition of the observed longitudinal field $(\mathrm{z}=0)$ in harmonics using the discrete Fast Fourier Transform. The method was first described by Alissandrakis (1981).

Figure 8 presents the results of the extrapolation overlying the EIT $195 \AA$ image at 07:13:30 UT, the EIT $304 \AA$ image at 06:24:11 UT, and MSDP $\mathrm{H} \alpha$ image at 06:15 UT. All are co-aligned with the MDI magnetogram at 06:34:11 UT. The last panel represents a 3D view of the extrapolated magnetic field lines in a box smaller than the computation box, to see better the connectivities. Note that since the magnetic field lines drawn in red in the central part of the region are not reliable, this area is strongly sheared due to the rotating emerging flux and the potential approximation is not appropriated, as we have mentioned above.

The magnetic field lines joining places out of the active regions are drawn in yellow. These field lines are rather arbitrary because of the effects of the periodicity of the method and because of the negative flux imbalance of the active region.

The green/dark blue lines correspond to loops connecting the secondary ribbon $R^{\prime} 1 / R^{\prime} 3$ to the eruptive area in the center of the active region. Though not observed, we consider that these large-scale and weakly sheared loops exist because of the continuous presence of the secondary ribbons. The light blue lines are associated to ribbon $\mathrm{R}^{\prime} 2$.

The results of the potential field extrapolation show what follows. The ribbons $\mathrm{R}^{\prime} 1$ and $\mathrm{R}^{\prime} 3$ visible in the EIT images and in $\mathrm{H} \alpha$ (MSDP) are well explained using this approach. The lines with the ends in these positive polarities and going towards the periphery of the region map well the loops directed toward the West and the South in the images of EIT/171 $\AA$ (see Figure 7 (e, f) and 8 $(\mathrm{a}, \mathrm{b}))$.

The pattern of field lines linked to $\mathrm{R}^{\prime} 2$ indicates that this zone is more eruptive than we expected because numerous lines connect to the main flare. Some blue lines are also linked to a small bright plage.

The overlying field lines indicate the possibility of the existence of a null point where some high altitude coronal reconnection could have occurred, i.e. in the zone between the red, dark blue and the yellow lines in the North of the active region (Figure 8, arrow). Indeed, the co-alignment with the H $\alpha$ MSDP image shows that the secondary ribbons are located at the footpoints of the lines that come from this zone. Different scenarios could explain the triggering of this high altitude reconnection and its manifestation as secondary remote ribbons. In case of a pre-brightening of the secondary ribbons before the flare, we could infer that we see a signature of the breakout model (Antiochos, DeVore, and Klimchuk, 
1999) as observed in some events (Aulanier et al., 2000; Mandrini et al., 2006). In the case of brightenings occurring at the same time as the flare, these could be a remanant of an arc-shaped Moreton wave or EIT wave surrounding the active region (Moreton, 1960; Thompson et al., 1998). The brightenings observed in our case seem to be only the stationary part of the EIT waves that Delannée, Hochedez, and Aulanier (2007) explained by Joule heating at the sites of QSLs. Balasubramaniam et al. (2005) described the Sequential Chromospheric Brightenings (SCB) that appear to be different from the Moreton/EIT waves. They explained these SCB as the footpoints of field lines that extend into the corona, where they are energized in sequence by magnetic reconnection as coronal fields tear from the chromosphere during the eruption.

\section{Conclusion}

We studied the M1.6 flare of May 27, 2003, occurring in the magnetically complex active region NOAA 10365. We used multi-wavelength observations, which include different instruments: the MSDP operating in the Meudon solar tower, RHESSI, TRACE, SOHO/EIT and SOHO/MDI.

The evolution of the photospheric magnetic configuration shows that the active region NOAA 10365 is characterized by the emergence of a twisted large magnetic flux tube. As the twisted flux is rising, diverging flows lead to a peculiar pattern of the photospheric longitudinal magnetic field. The elongated polarities, positive and negative, indicate that a right hand twisted flux tube emerges exhibiting two elongated "tongues" (López Fuentes et al., 2000). This implies to an increase of positive helicity, as also noticed by Chae, Moon, and Park (2004).

In $\mathrm{H} \alpha$ as well as in TRACE $1600 \AA$ we observe a double ' $\mathrm{J}$ ' shaped ribbon distribution. This corresponds to a positive magnetic helicity signature. The asymmetric sheared distribution of the non-thermal HXR sources observed by RHESSI in the 25-50 keV range also confirms this result. It is the first time that we have been able to find circumstantial evidence for the same sign of helicity in the flux emergence and the eruption signatures, although it has already been predicted that sunspot whorls, tongues, 'J'-shaped ribbons or sigmoids are signatures of magnetic helicity of the same sign according to their shape (see the review by Démoulin and Pariat, 2009).

Regarding secondary arc-shaped ribbons, observed at the periphery of the active region, we are able to correlate them with separatrices found in potential field extrapolation using the code from the database FROMAGE.

Two scenarios are possible to explain the brightenings of the secondary ribbons: a breakout of the overlying magnetic field lines before the main flare as Antiochos, DeVore, and Klimchuk (1999) proposed, and/or some compression and ohmic dissipation produced in separatrices during the flare, as proposed by Delannée, Hochedez, and Aulanier (2007) to explain the components of Moreton/EUV waves. Unfortunately, the lack of high cadence observations with SOHO/EIT and the limited field of view of TRACE do not allow to conclude if either scenario indeed applies here. Future high cadence and full disc EUV images from the upcoming SDO mission should be well suited to address this issue. 
Acknowledgements The authors thank Arnaud Bérurier who provided all the analysis of this event during a four months of training in solar physics, Dr. Pascal Démoulin for fruitful discussions and Dr. Säm Krucker for his advices in the RHESSI data processing. We also thank the anonymous referee for his/her constructive comments and suggestions, which improved the paper significantly. R.C. thanks the CEFIPRA for his post doc grant. Financial support by the European Commission through the SOLAIRE Network (MTRN-CT-2006-035484) is gratefully acknowledged.

\section{References}

Alissandrakis, C.E.: 1981, Astron. Astrophys. 100, 197-200.

Antiochos, S.K., DeVore, C.R., Klimchuk, J.A.: 1999, Astrophys. J. 510, $485-493$.

Aulanier, G., DeLuca, E.E., Antiochos, S.K., McMullen, R.A., Golub, L.: 2000, Astrophys. J. $\mathbf{5 4 0}, 1126-1142$.

Aulanier, G., Golub, L., DeLuca, E.E., Cirtain, J.W., Kano, R., Lundquist, L.L., Narukage, N., Sakao, T., Weber, M.A.: 2007, Science 318, 1588-1591.

Balasubramaniam, K.S., Pevtsov, A.A., Neidig, D.F., Cliver, E.W., Thompson, B.J., Young, C.A., Martin, S.F., Kiplinger, A.: 2005, Astrophys. J. 630, 1160-1167.

Berger, M.A.: 1984, Geophys. and Astrophys. Fluid Dyn. 30, 79-104.

Berlicki, A., Schmieder, B., Vilmer, N., Aulanier, G., Del Zanna, G.: 2004, Astron. Astrophys. 423, $1119-1131$

Brueckner, G.E., Howard, R.A., Koomen, M.J., Korendyke, C.M., Michels, D.J., Moses, J.D., Socker, D.G., Dere, K.P., Lamy, P.L., Llebaria, A., Bout, M.V., Schwenn, R., Simnett, G.M., Bedford, D.K., Eyles, C.J.: 1995, Solar Phys. 162, 357-402.

Chae, J., Moon, Y.J., Park, Y.D.: 2004, Solar Phys. 223, 39-55.

Chandra R., Pariat E., Schmieder B., Mandrini C.H., Uddin W.: 2009, Solar Phys.(submitted).

Chen, P.F., Shibata, K.: 2000, Astrophys. J. 545, 524-531.

Choudhary, D.P., Ambastha, A., Ai, G.: 1998, Solar Phys. 179, 133-140.

Delaboudinière, J.P., Artzner, G.E., Brunaud, J., Gabriel, A.H., Hochedez, J.F., Millier, F., Song, X.Y., Au, B., Dere, K.P., Howard, R.A., Kreplin, R., Michels, D.J., Moses, J.D., Defise, J.M., Jamar, C., Rochus, P., Chauvineau, J.P., Marioge, J.P., Catura, R.C., Lemen, J.R., Shing, L., Stern, R.A., Gurman, J.B., Neupert, W.M., Maucherat, A. Clette, F., Cugnon, P., van Dessel, E.L.: 1995, Solar Phys. 162, $291-312$.

Delannée, C., Hochedez, J.F., Aulanier, G.: 2007, Astron. Astrophys. 465, 603-612.

Démoulin, P.: 2007, Adv. Space Res. 39, 1674-1693.

Démoulin, P., Bagala, L.G., Mandrini, C.H., Henoux, J.C., Rovira, M.G.: 1997, Astron. Astrophys. 325, 305-317.

Démoulin, P., Henoux, J.C., Priest, E.R., Mandrini, C.H.: 1996, Astron. Astrophys. 308 $643-655$.

Démoulin, P., Pariat, E.: 2009, Adv. Space Res. 43, $1013-1031$.

Démoulin, P., Priest, E.R., Lonie, D.P.: 1996, J. Geophys. Res. 101, $7631-7646$.

Green, L.M., Kliem, B., Török, T., van Driel-Gesztelyi, L., Attrill, G.D.R.: 2007, Solar Phys. 246, 365-391.

Hagyard, M.J., Venkatakrishnan, P., Smith, J.B. Jr.: 1990, Astrophys. J. Suppl. Ser. 73, $159-163$.

Handy, B.N., Acton, L.W., Kankelborg, C.C., Wolfson, C.J., Akin, D.J., Bruner, M.E., Caravalho, R., Catura, R.C., Chevalier, R., Duncan, D.W., Edwards, C.G., Feinstein, C.N., Freeland, S.L., Friedlaender, F.M., Hoffmann, C.H., Hurlburt, N.E., Jurcevich, B.K. Katz, N.L., Kelly, G.A., Lemen, J.R., Levay, M., Lindgren, R.W., Mathur, D.P., Meyer, S.B., Morrison, S.J., Morrison, M.D., Nightingale, R.W., Pope, T.P., Rehse, R.A. Schrijver, C.J., Shine, R.A., Shing, L., Strong, K.T., Tarbell, T.D., Title, A.M., Torgerson, D.D., Golub, L., Bookbinder, J.A., Caldwell, D., Cheimets, P.N., Davis, W.N., Deluca, E.E., McMullen, R.A., Warren, H.P., Amato, D., Fisher, R., Maldonado, H., Parkinson, C.: 1999, Solar Phys. 187, 229-260.

Heyvaerts, J., Priest, E.R., Rust, D.M.: 1977, Astrophys. J. 216, 123-137. 
Hurford, G.J., Schmahl, E.J., Schwartz, R.A., Conway, A.J., Aschwanden, M.J., Csillaghy, A., Dennis, B.R., Johns-Krull, C., Krucker, S., Lin, R.P., McTiernan, J., Metcalf, T.R., Sato, J., Smith, D.M.: 2002, Solar Phys. 210, 61-86.

LaBonte, B.J., Georgoulis, M.K., Rust, D.M.: 2007, Astrophys. J. 671, 955-963.

Leka, K.D., Canfield, R.C., McClymont, A.N., van Driel-Gesztelyi, L.: 1996, Astrophys. J. 462, $547-560$.

Li, H., Schmieder, B., Song, M.T., Bommier, V.: 2007, Astron. Astrophys. 475, 1081-1091.

Lin, R.P., Dennis, B.R., Hurford, G.J., Smith, D.M., Zehnder, A., Harvey, P.R., Curtis, D.W. Pankow, D., Turin, P., Bester, M., Csillaghy, A., Lewis, M., Madden, N., van Beek, H.F., Appleby, M., Raudorf, T., McTiernan, J., Ramaty, R., Schmahl, E., Schwartz, R., Krucker, S., Abiad, R., Quinn, T., Berg, P., Hashii, M., Sterling, R., Jackson, R., Pratt, R., Campbell, R.D., Malone, D., Landis, D., Barrington-Leigh, C.P., Slassi-Sennou, S., Cork, C., Clark, D., Amato, D., Orwig, L., Boyle, R., Banks, I.S., Shirey, K., Tolbert, A.K. Zarro, D., Snow, F., Thomsen, K., Henneck, R., McHedlishvili, A., Ming, P., Fivian, M., Jordan, J., Wanner, R., Crubb, J., Preble, J., Matranga, M., Benz, A., Hudson, H., Canfield, R.C., Holman, G.D., Crannell, C., Kosugi, T., Emslie, A.G., Vilmer, N., Brown, J.C., Johns-Krull, C., Aschwanden, M., Metcalf, T., Conway, A.: 2002, Solar Phys. 210, 3-32.

López Fuentes, M.C., Démoulin, P., Mandrini, C.H., van Driel-Gesztelyi, L.: 2000, Astrophys. J. 544, 540-549.

Mandrini, C.H., Démoulin, P., Schmieder, B., Deluca, E.E., Pariat, E., Uddin, W.: 2006, Solar Phys. 238, 293-312.

Martin, S.F., Dezso, L., Antalova, A., Kucera, A., Harvey, K.L.: 1982, Adv. Space Res. 2, $39-51$.

Mason, D., Komm, R., Hill, F., Howe, R., Haber, D., Hindman, B.W.: 2006, Astrophys. J. 645, $1543-1553$.

Mein, P.: 1977, Solar Phys. 54, 45-51.

Mein, P.: 1991, Astron. Astrophys. 248, 669-676.

Moore, R.L., Larosa, T.N., Orwig, L.E.: 1995, Astrophys. J. 438, 985-966.

Moreton, G.E.: 1960, Astronom. J. 65, 494-495.

Pariat, E., Nindos, A., Démoulin, P., Berger, M.A.: 2006, Astron. Astrophys. 452, 623-630.

Pevtsov A.A.: 2002, In: Martens, P.C.H., Cauffman, D. (eds.) Multi-Wavelength Observations of Coronal Structure and Dynamics., 125-134.

Pevtsov, A.A., Canfield, R.C., Metcalf, T.R.: 1995, Astrophys. J. 440, L109-L112.

Priest, E.R., Démoulin, P.: 1995, J. Geophys. Res. 100, 23443-23464.

Roudier, T., Švanda, M., Meunier, N., Keil, S., Rieutord, M., Malherbe, J.M., Rondi, S., Molodij, G., Bommier, V., Schmieder, B.: 2008, Astron. Astrophys. 480, 255-263.

Sammis, I., Tang, F., Zirin, H.: 2000, Astrophys. J. 540, 583-587.

Scherrer, P.H., Bogart, R.S., Bush, R.I., Hoeksema, J.T., Kosovichev, A.G., Schou, J., Rosenberg, W., Springer, L., Tarbell, T.D., Title, A., Wolfson, C.J., Zayer, I., MDI Engineering Team, : 1995, Solar Phys. 162, 129-188.

Schmieder, B., Démoulin, P., Aulanier, G., Golub, L.: 1996, Astrophys. J. 467, 881-886.

Schmieder, B., Hagyard, M.J., Guoxiang, A., Hongqi, Z., Kalman, B., Gyori, L., Rompolt, B., Demoulin, P., Machado, M.E.: 1994, Solar Phys. 150, 199-219.

Schmieder, B., van Driel-Gesztelyi L.: 2005, In: Dere, K., Wang, J., Yan, Y. (eds.) Coronal and Stellar Mass Ejections, IAU Symp., 226, $149-160$.

Shibata, K., Nozawa, S., Matsumoto, R.: 1992, Pub. Astron. Soc. Japan 44, 265-272.

Thompson, B.J., Plunkett, S.P., Gurman, J.B., Newmark, J.S., St. Cyr, O.C., Michels, D.J.: 1998, Geophys. Res. Lett. 25, 2465-2468.

Williams, D.R., Török, T., Démoulin, P., van Driel-Gesztelyi, L., Kliem, B.: 2005, Astrophys. J. 628, L163-L166.

Zhang, Y., Zhang, M., Zhang, H.: 2008, Solar Phys. 250, 75-88.

Zhao, J., Kosovichev, A.G.: 2004, Astrophys. J. 603, 776-784. 
ms_27may03.tex; 10/09/2018; 2:24; p.18 